Musées, Patrimoine et Culture scientifiques et techniques

$129 \mid 2010$

mai - juin 2010

\title{
Les universités doivent-elles vraiment exposer leurs collections au grand public?
}

Should universities really exhibit their collections to the general public?

\section{Michèle Antoine}

\section{OpenEdition \\ Journals}

Édition électronique

URL : http://journals.openedition.org/ocim/149

DOI : 10.4000/ocim. 149

ISSN : 2108-646X

Éditeur

OCIM

Édition imprimée

Date de publication : 1 mai 2010

Pagination : 7-12

ISSN : 0994-1908

Référence électronique

Michèle Antoine, "Les universités doivent-elles vraiment exposer leurs collections au grand public ? ", La Lettre de I'OCIM [En ligne], 129 | 2010, mis en ligne le 01 mai 2012, consulté le 01 mai 2019. URL http://journals.openedition.org/ocim/149; DOI : 10.4000/ocim.149 
qualité reconnue, destiné au grand public et « relativement accessible » pour un musée universitaire (soit uniquement l'après midi en semaine), à des collections totalement anecdotiques (celle d'anthropologie physique par exemple) uniquement consultables sur demande. La question posée de manière sous-jacente était - et je la reformule - : « comment exister vis-à-vis $d u$ grand public dans une ville dans laquelle on trouve pas moins de 80 musées, et parmi eux quatre grands musées fédéraux qui bénéficient de collections et de budgets d'une taille totalement incomparables à ceux de l'université? » (1)

Cette première expérience a piqué ma curiosité. Elle m'a amenée à m’interroger, au gré des rencontres et des visites, à propos de la diffusion des collections universitaires et à relever quelques impasses intellectuelles souvent associées à cette question.

Les musées et collections universitaires existent en grand nombre de part le monde, et toutes ont un visage différent. Certaines collections sont d'importance nationale et appartiennent de fait au monde des musées « classiques », il est clair que leurs responsables ne se reconnaîtront pas dans ce qui suit. Mais à ma connaissance beaucoup de collections universitaires partagent un ensemble de caractéristiques :

- Les universités possèdent souvent un grand nombre de collections disparates, appartenant à des domaines académiques variés et de qualité très inégale. Cette hétérogénéité intrinsèque provient bien souvent de la manière dont ces collections ont été rassemblées au cours du temps. Certaines sont constituées en fonction d'un intérêt spécifique et souvent circonstancié d'un ou plusieurs chercheurs (domaine de recherche justifiant la constitution d'une collection par exemple). D’autres sont acquises dans un but pédagogique lorsque l'objet réel ou sa reproduction fidèle est indispensable à l'étude (en Zoologie, en Botanique ou en Anatomie, par exemple). Tandis que d'autres encore résultent de donations ou legs parfois sans rapport avec l'activité universitaire.

- La plupart de ces collections sont rassemblées dans quelques bâtiments ou locaux, difficilement accessibles, parfois ouverts uniquement durant les périodes de cours. Cette restriction ne dérive pas d'une quelconque volonté délibérée de lier les collections aux objectifs pédagogiques mais bien de réels problèmes organisationnels. Il est vrai qu'ouvrir ses portes au grand public signifie lui donner accès durant les périodes où il est susceptible de visiter les collections c'est-à-dire - hormis pour le scolaire après les heures de travail et durant les week-ends.
Cela impose l'engagement de personnels surnuméraires, ce qui est rapidement très lourd en terme financier et difficilement supportable pour une institution dont ce n'est pas la priorité.

- Leur public est souvent très restreint. Quelques chercheurs, quelques amateurs éclairés y viennent sur une base volontaire, les étudiants y sont parfois amenés dans le cadre de leurs cours, de façon quasi

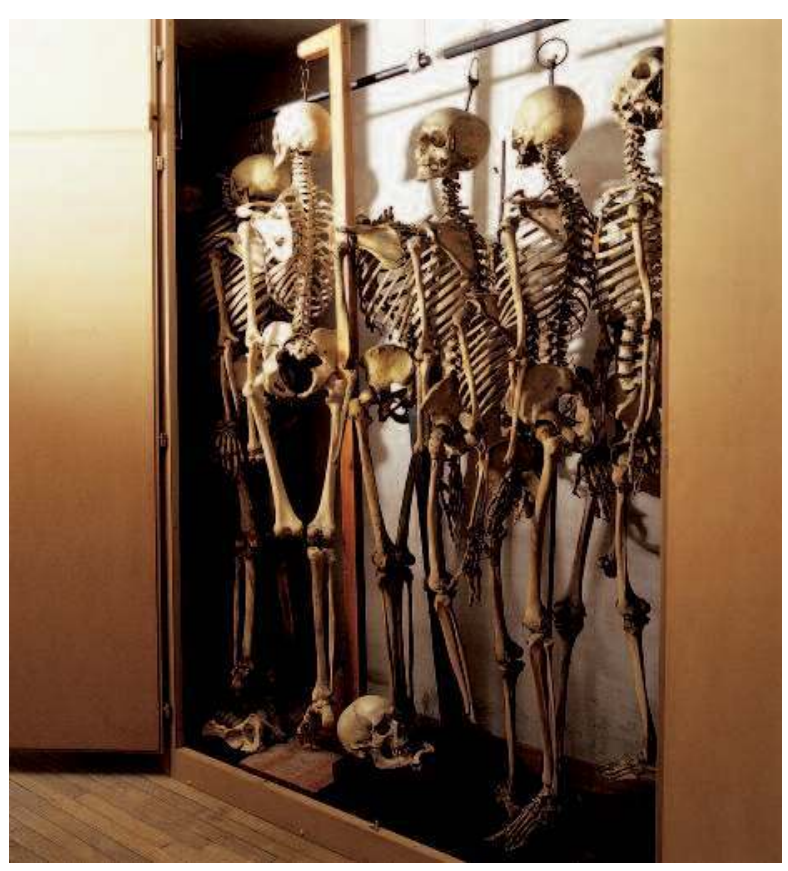

Armoire de rangement des squelettes, collection de l'institut d'Anatomie normale, université de Strasbourg (c) Université de Strasbourg

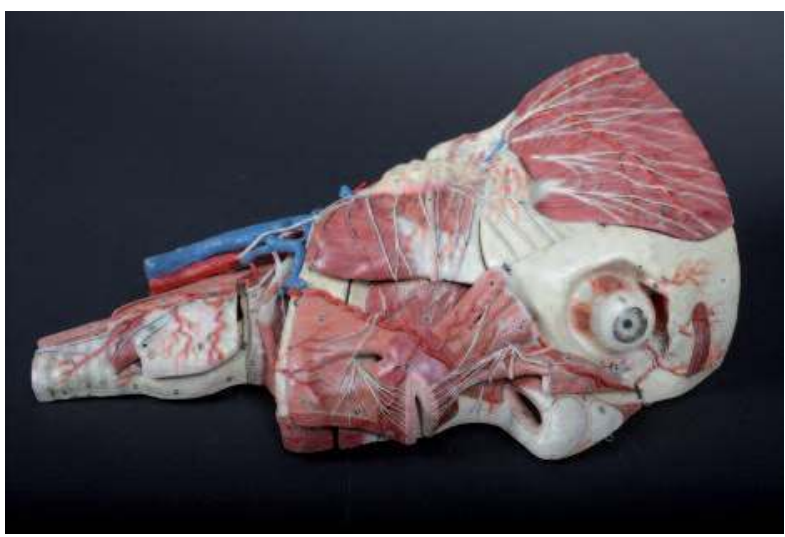

Modèle pédagogique, collection d'Anatomie, université de Bourgogne () Vincent Arbelet 
obligatoire. Pourtant, dans le discours, ces étudiants semblent constituer la première cible de la diffusion des collections universitaires.

Malgré cet état de fait constaté par beaucoup, de nombreuses universités proclament leur ambition de toucher un large public, universitaire et non universitaire, en créant une institution plus ou moins unifiée ${ }^{(2)}$. Les raisons qui les poussent sont d'ailleurs très diverses selon la position institutionnelle du porteur de projet :

- Du côté des académiques, la motivation principale est pédagogique. Il s'agit de diffuser la connaissance générée dans l'institution : la connaissance relative aux objets collectionnés, corpus qui, grâce à des études récentes, tend à s'accroître, mais également la connaissance liée à la recherche effectuée dans l'université. Cette démarche s'inscrit aussi dans le prolongement de la tâche d'enseignement de ces institutions. On évoque souvent, surtout dans le domaine des sciences exactes, la nécessité d'attirer vers des domaines délaissés par les étudiants mais également la nécessité d'éclairer le citoyen par rapport à des matières qui font débat (OGM, Espace...). Créer un «musée universitaire » ressortirait donc de la responsabilité sociale de l'université. Il est à noter que cet objectif est particulièrement sensible dans les villes universitaires où l'on ne retrouve pas de musées ou de centres d'exposition assurant cette diffusion de la connaissance scientifique.

- Pour les instances dirigeantes, il s'agit plutôt de promouvoir l'université, de valoriser son image dans ce qui est en train de devenir à la fois un marché de l'enseignement (songeons aux conséquences du processus de Bologne sur la mobilité des étudiants et donc la possibilité de choisir entre des universités) et un marché de la recherche où l'université doit capter l'attention des partenaires financiers publics et privés. Un musée n'est-il pas la vitrine rêvée des activités présentes (ou passées) de l'institution ? Créer un «musée universitaire » ferait donc partie de la stratégie de séduction de l'université.

- Les responsables des collections, enfin, qui souvent travaillent en solitaire, dans l'ombre depuis de nombreuses années, trouvent dans un projet de musée universitaire - même s'il reste à l'état de projet d'ailleurs la possibilité de mobiliser attention et ressources supplémentaires en faveur d'une meilleure conservation des collections. C'est souvent également l'occasion de donner une impulsion au processus d'inventorisation, de documentation et de catalogage des collections. «Créer un musée universitaire » deviendrait avant tout un acte de sauvegarde du patrimoine.
Ces trois descriptions sont à dessein de l'ordre de la caricature. Les différents projets qui ont émergé ces dernières années en Europe occidentale mêlent souvent les trois aspects avec une prédominance de l'un ou l'autre selon les cas. Mais si beaucoup de projets ont fait l'objet d'annonces, peu ont réellement été développés. Nonobstant les aspects de politique locale ou universitaire qui peuvent avoir plombé leur réussite, plusieurs causes internes peuvent être identifiées.

Un premier problème découle de la difficulté pour ces projets de trouver un sujet cohérent qui pourrait constituer la base d'une institution reconnaissable de l'extérieur. Souvent, la question est traitée de manière « internaliste »; je veux dire en examinant les principales caractéristiques des collections et en essayant de trouver une ligne cohérente entre elles. Cela conduit quasi inévitablement à créer un musée des sciences (ou de la connaissance) lato sensu. Par la définition de cette coupole très large, on espère intégrer des groupes d'objets très hétérogènes mais surtout on prétend refléter l'activité de recherche de l'université. Cependant le fossé est tellement large entre les objets, leurs significations intrinsèques, leurs contextes de récolte... et le travail scientifique réellement effectué dans ces institutions en ce début de XXI e siècle qu'une telle approche s'avère souvent impraticable. De plus, les collections universitaires couvrent rarement tous les domaines du savoir scientifique. Tout comme les recherches effectuées par une université sont généralement partielles et éclatées. Et il est rare que les richesses des unes concordent aux points forts des autres.

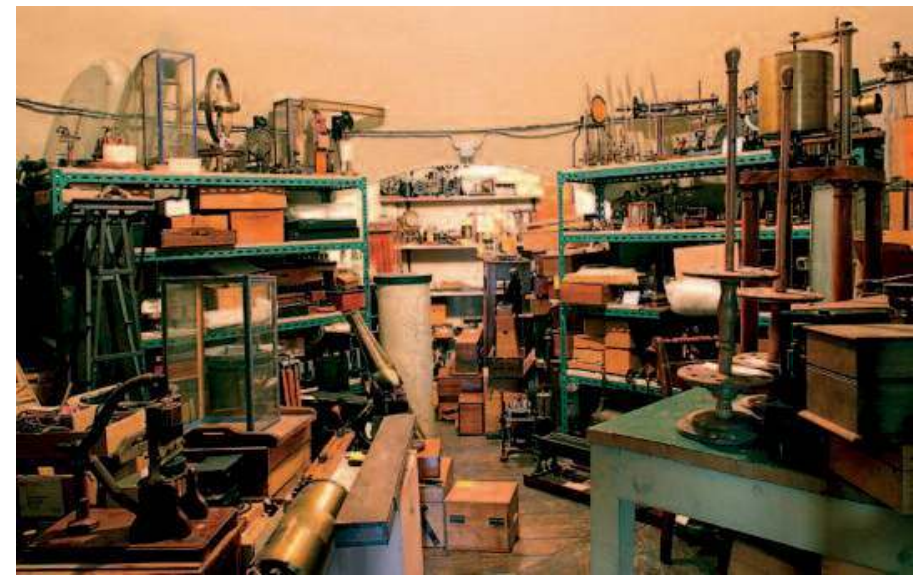

Réserve des instruments scientifiques à l'université de Strasbourg (๐) Service régional de l'inventaire/université de Strasbourg/Claude Menninger 
Un second obstacle est lié à la qualité, souvent toute relative, des collections concernées. Le mode d'acquisition des collections, souvent erratique, déjà évoqué plus haut, et la faiblesse des ressources financières attribuées par les universités rendent les acquisitions volontaires très rares. Il est donc impossible de construire qualitativement la collection et il faut s'en remettre au hasard pour que des pièces de qualité scientifique et patrimoniale indiscutable s'inscrivent dans le patrimoine d'une université. Mais la situation est encore plus délicate quand on passe dans le domaine de la diffusion à un public non spécialisé. C'est alors que l'on découvre qu'un objet de grande valeur scientifique ou de grand intérêt patrimonial peut être totalement indécodable pour le commun des mortels, sauf à y ajouter un tel nombre de couches d'interprétation qu'il disparaît de fait et que son intérêt comme source de médiation devient inexistant. La difficile tâche de médiation fait comprendre qu'un objet patrimonial n'est pas nécessairement un objet muséal. Pour être considéré comme tel, un objet doit être aisément « lisible » pour le visiteur, il doit être porteur d'une histoire à raconter (ou d'un phénomène à démontrer) et il doit générer une émotion (parce qu'il est beau, parce quili est ancien, parce quili a appartenu à quelqu'un de célèbre...). Même pour les musées dits classiques, le défi est grand mais ils disposent, eux, de moyens d'acquérir quelques-uns de ces objets qui deviennent les éléments phares de leurs expositions.

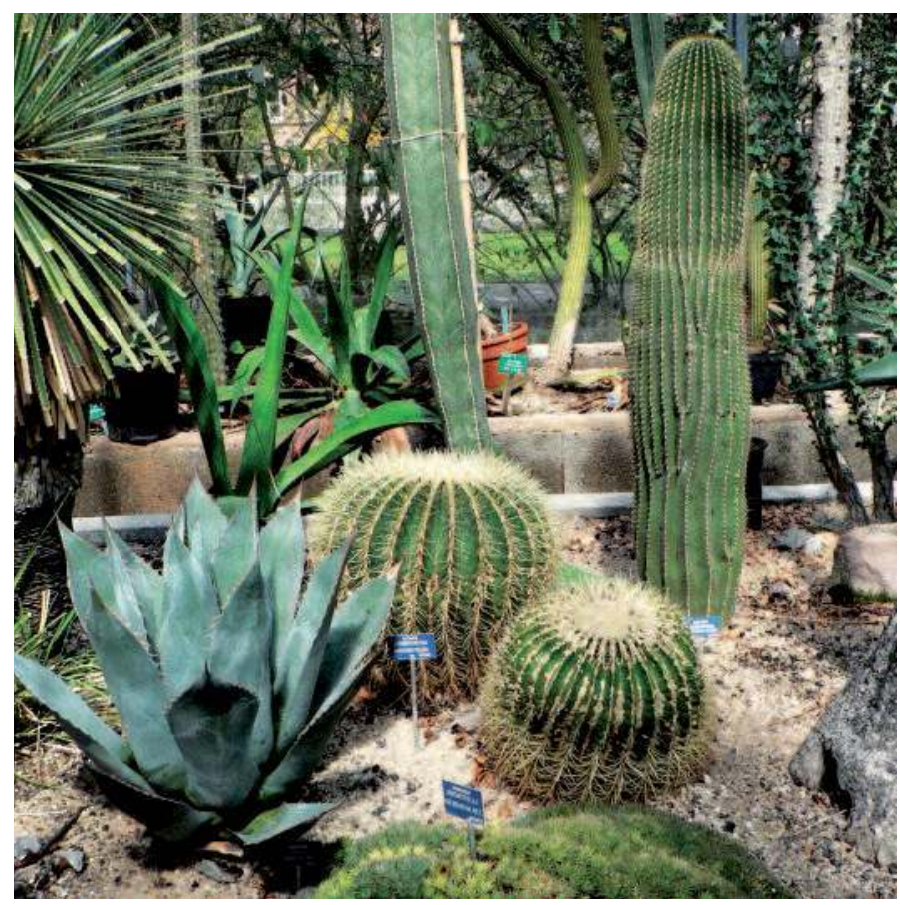

Le jardin botanique de l'université de Strasbourg ○) université de Strasbourg/Étienne Haouy
À cette difficulté de donner de leur contenu une image simple et claire pour le grand public, et à la fréquente pénurie d'objets de qualité muséale, s'ajoute souvent un manque de personnel dédié et qualifié et des ressources financières insuffisantes. Les musées et collections universitaires sont donc bien mal armés pour s'inscrire dans le marché des musées, ou des autres activités liées à l'edutainment (3), qui sont pourtant leurs concurrents dans les choix d'activités de loisirs du grand public... et du public étudiant.

On voit donc bien que la question qui mérite d'être posée n'est pas seulement celle du « quoi » ou « que » présenter. Il faut également se demander " comment», « pour qui ", « dans quel contexte » et surtout « dans quel but » préserver les collections universitaires.

Au vu des obstacles pointés précédemment, il me semblerait opportun de considérer la question des musées universitaires sous un autre angle, selon une démarche que l'on pourrait qualifier de marketing, c'est-à-dire en tenant compte de la concurrence et en essayant d'identifier de nouvelles niches originales dans lesquelles les musées des universités pourraient faire la différence. Je propose donc de s'interroger sur ce que pourrait faire un musée universitaire qui lui soit totalement spécifique et pour lequel il aurait une pleine légitimité et une totale expertise. En d'autres termes, que pourrait faire un musée universitaire que les autres musées (ou institutions similaires) ne font pas?

Je voudrais proposer trois pistes ${ }^{(4)}$ :

- mettre en scène un moment iconique de l'histoire de l'université ;

- expliquer, démontrer, faire vivre la méthode scientifique ;

- devenir un lieu d'expérimentation muséologique.

\section{Un moment iconique de l'histoire de l'université}

Les universités ont une histoire, parfois très longue, bien souvent ignorée du grand public, des étudiants et du personnel de l'université. Or cette histoire spécifique présente généralement des liens avec des éléments d'histoire des sciences, ou avec l'histoire du développement de la cité. Un musée universitaire, pour peu que ses collections comprennent des objets s'y rapportant, peut alors devenir un lieu unique où cette histoire est racontée, en toute légitimité. 


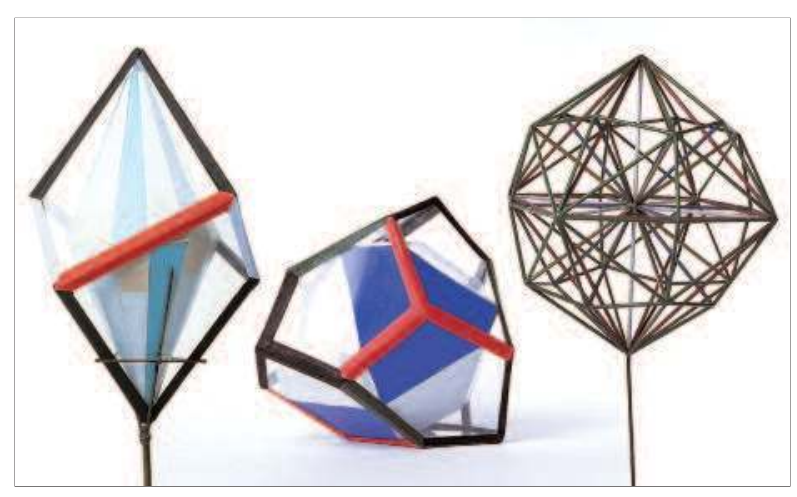

Modèle pédagogique, collection de Minéralogie, université de Strasbourg () Université de Strasbourg

Prenons un exemple dans une très grande institution The Royal Institution of Great Britain. Celle-ci possède de nombreux objets et archives appartenant à Faraday. Elle dispose même de son laboratoire. Elle a donc concentré son exposition sur la vie de ce savant et sur son influence dans l'histoire de la Physique (électricité). Partant de ce point de référence, elle a pu déborder pour parler d'autres sujets liés à l'électrophysique. Un tel choix donne automatiquement une légitimité à la présentation des collections et une vraie raison pour visiter le musée, car s'y joue l'émotion de confronter le vrai, l'authentique, l'unique, toutes choses que recherche le visiteur de musée : le lieu où Faraday a travaillé. La logique en œuvre devient celle des maisons historiques, ou centres d'interprétation, très populaires dans le monde des musées.

\section{La méthode scientifique}

Malgré bien des ambitions, la méthode scientifique est souvent le grand absent du musée de science et encore plus des musées de sciences humaines l'histoire ou l'épistémologie des sciences sont rarement expliquées. Pourquoi ? Parce que les musées classiques privilégient le "wouaw ", l'émerveillement. Ils présentent de beaux objets, racontent des histoires, expliquent des phénomènes, vulgarisent des connaissances mais ils oublient souvent d'expliquer comment ces connaissances ont été construites, comment ceux qui les ont élaborées pensent, travaillent... La raison sous jacente à cet état de fait ne provient pas uniquement de la nécessité de «plaire » aux visiteurs. Elle résulte du fait que dans de nombreux cas, les scientifiques, je veux dire les chercheurs, ceux qui font la science, ne sont pas présents dans l'institution. Le travail de diffusion des connaissances et des collections repose sur ce

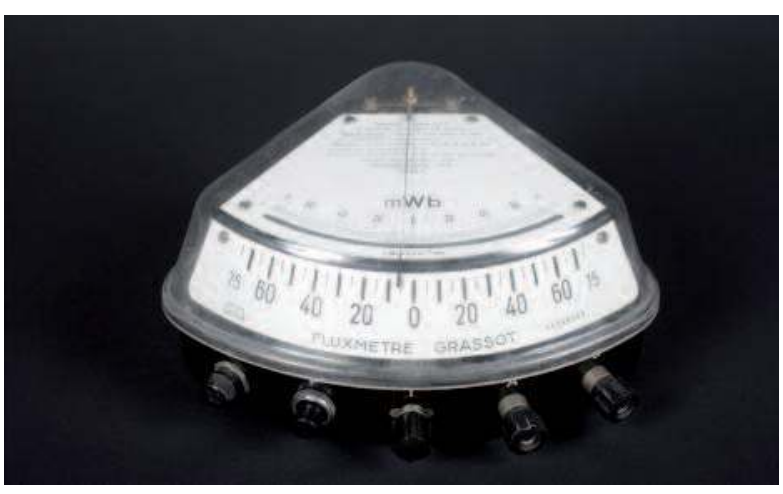

Fluxmètre, collection de Physique, université de Bourgogne () Vincent Arbelet

qu'ils écrivent et non pas sur ce qu'ils font. Cela aussi pourrait, par contraste, constituer pour l'université, l'occasion de faire quelque chose d'unique, de différent. La chance de l'université est d'être le lieu où le savoir est construit. Elle est donc totalement légitime pour prendre en charge cet aspect de la (méta)connaissance, en mettant en scène le travail quotidien, les expérimentations, les essais, erreurs, découvertes et échecs qui sont la substance même du travail scientifique réel (et non idéalisé). Instruments historiques ou spécimens anciens pourraient d'ailleurs être utilisés pour ce type de présentation car ils sont plus évidents à comprendre : pas de boîte noire, mais des expériences réelles, où le processus hypothético-déductif peut être mis plus aisément en évidence.

\section{Expérimentation}

Mais s'il est une chose pour laquelle les universités semblent faites, c'est bien pour l'expérimentation, l'innovation, la fertilisation croisée des idées. Plutôt que de se focaliser sur les collections ou les savoirs à vulgariser - déjà pris en charges par d'autres - elles pourraient créer de véritables laboratoires qui partent des objets et des idées.

Quelques exemples permettent d'illustrer mon propos. À l'université Laval (Québec) un laboratoire a été mis en place pour étudier de nouvelles manières d'exposer les objets, de la muséographie expérimentale (5). Le LAMIC, dirigé par Philippe Dubé étudie la méthode de transmission de la culture à travers l'expérience muséale. Ses travaux se concentrent sur trois domaines de la recherche fondamentale en muséologie : l'espace, l'objet et le visiteur. Pour chaque champ, les chercheurs s'engagent dans des expériences soit dans le monde réel (numérisation 
3D, développement d'espaces d'exposition, technologie intelligente d'observation du visiteur), soit dans le monde virtuel (modélisation, transfert d'expérience en temps réel, montages visuels complexes). Ces travaux nécessitent la présence, l'interaction avec des visiteurs qui peuvent trouver dans la démarche d'expérimentation de nouvelles expériences à vivre.

Un autre type d'expérimentation peut également provenir de la manière dont l'université s'attache à croiser les expertises dans des domaines différents. Un bon exemple de cette approche est donné par la Science Gallery développée dans le campus du Trinity College à Dublin. Des questions scientifiques d'actualité, souvent très pointues, sont développées grâce à la collaboration étroite entre artistes, scientifiques et étudiants (d'ailleurs devenus «membres de la Galerie » dans le cadre d'ateliers dont le résultat fait l'objet d'exposition). Se mettent ainsi en place une nouvelle façon de parler de science mais également, pour les étudiants, une nouvelle manière de vivre un musée comme étant un lieu qui leur est ouvert et où la convivialité et l'échange sont privilégiés. Ce dernier aspect est renforcé par la présence au sein de la galerie d'un café-lounge très fréquenté par la communauté universitaire.

Ces quelques pistes ont bien entendu une valeur essentiellement exploratoire. Il serait vain de penser qu'elles constituent la panacée, la voie à suivre pour que les musées universitaires réussissent leur rencontre avec le grand public, voire avec le public universitaire. Mon propos est bien plus de pousser les responsables des musées et collections universitaires à réfléchir à leur originalité, à leur spécificité, à sortir des sentiers battus, pour découvrir d'autres voies de diffusion de leurs collections. Exemptées des impératifs d'audimat qui trop souvent et de plus en plus conditionnent le travail des musées classiques et orientent leur programmation et leur mise en exposition, les universités sont en position de chercher et d'innover dans ce domaine. Les bibliothèques universitaires - et je pense à celle de l'université d'Angers - ont ouvert la voie en repensant leur rôle non plus en terme de conservation de livres mis à disposition mais bien comme lieu de vie (intellectuelle) et de mise en réseau au service des usagers universitaires. Le pas était immense à franchir pour elle, pourquoi les musées universitaires ne pourraient-ils par en faire autant?

\section{Notes}

(1) Contre l'avis de certains experts, dont j'étais, l'université libre de Bruxelles y répondit en créant un réseau qui rassemble ses musées et collections, espérant ainsi créer une masse critique qui augmenterait sa visibilité et donc ses capacités promotionnelles. Le temps et l'expérience ont montré que cette solution n'avait apporté aucune amélioration.

(2) Soubiran, S., Lourenço, M.-C., Wittje, R., Talas, S. et Bremer, T. Initiatives européennes et patrimoine universitaire, la Lettre de l'OCIM $n^{\circ} 123$, mai-juin 2009, pp. 5-14.

(3) Edutainment vient de la contraction des mots anglais education et entertainment. Il désigne un certain type d'activités caractéristiques des musées notamment, et particulièrement des musées et centres de sciences, qui visent à faire apprendre en s'amusant. Parfois traduit sous les termes activités ludo-éducatives.

(4) Notons qu'une quatrième piste mériterait d'être explorée qui reposerait sur des collaborations entre des collections universitaires et des musées déjà bien implantés, les premiers se concentrant sur la gestion des collections et leur mise à disposition pour les chercheurs, les seconds se chargeant de les mettre en valeur. Il faut toutefois reconnaître que cette piste, qui correspond à une gestion rationnelle des compétences et des ressources, ne permet pas toujours d'atteindre l'objectif de valorisation de l'image de l'université.

(5) Dubé, P. La mise en laboratoire du musée, la Lettre de l'OCIM, n¹06, juillet-août 2006, pp. 11-17. 